\title{
Can point-of-care lung ultrasound (POCUS) be implemented as a substitute for chest CT scan for the assessment of patients with COVID-19 pneumonia? Treefa Salih Hasan (MBChB, DMRD, FRCR) ${ }^{1}$
}

\author{
${ }^{1}$ Department of radiology, Erbil Teaching Hospital, Erbil ,Iraq \\ Correspondence Address: \\ Treefa Salih Hasan \\ Department of radiology, Erbil Teaching Hospital, Erbil ,Iraq \\ email: trefa_77@yahoo.com
}

Received: 22 November 2020

Revised: 15 December 2020

Accepted: 17 January 2021

Published: 25 June 2021

Diyala Medical Journal 2021:20(2): 43- 55

\section{Abstract}

Background: In the novel COVID outbreak, chest imaging was in the front door for the diagnostic approach to any patient with respiratory symptoms, including chest HRCT \& lung ultrasound modalities.

Objective: To find the correlation between lung ultrasound and chest high-resolution computed tomography (HRCT) signs in patients suffering from COVID-19 pulmonary involvement.

Patients and Methods: This cross-sectional observational study conducted in Arbil / Iraq, from July to November 2020 , enrolled 50 patients who assessed using HRCT and lung ultrasound (LUS), 12 areas in the chest examined by ultrasound and corresponding percentage (absent or limited, moderate, extensive, severe \& critical grads ) of lung affection on HRCT. Results: Analysis of the obtained data from 50 affected patients done, a highly significant positive relation noted between the findings on ultrasound \& the extend of lung involvement by chest HRCT ( $r$ factor 0.78 , the p-value was $<0.001$ ), the sensitivity, specificity, and accuracy of LUS were $90 \%, 70 \%$, and $68 \%$ respectively.

Conclusion: The current study showed that ultrasound and HRCT are parallel in assessing the extent of lung involvement among COVID-19 pneumonia victims, thus making lung ultrasound a relevant substitute for HRCT.

Keywords: lung ultrasound ; chest CT ; COVID-19 


\section{Introduction}

In the context of recently emerged COVID19 respiratory infection which began in Yohan followed by global spread ,the greatest requirement for chest imaging raised , advanced studies have elicited that chest high resolution computed tomography (HRCT) is paramount not only for primary diagnosis ,but also for screening and evaluation of COVID-19 pneumonia severity [1]). HRCT is much more sensitive in COVID-19 diagnosis than (RT-PCR) [2].

Considering the high fatality rate of patients with COVID-19, the need for transporting \& moving contagious patients between the hospital departments for CT exam rendered the situation dangerous [3]. Nevertheless, on basis of limiting the spread of infection, bed-side ultrasonography is a feasible substitute, after securing proper equipment sterilizing and protection.

From the early 2000s, a modern trend for lung ultrasound (LUS) implementation has been introduced to assess pulmonary parenchymal disease in addition to pleural effusions and masses lesions [2].

Normal pleura elicit hyperechoic horizontal line when the underlying lung is healthy, deep to which creates repetitive parallel similar thin horizontal lines known as Alines Figure (1) ,in the diseased lung, the lung air replaced, in turn leads to reduced acoustic impedance difference due to closer densities in lung contents and lung tissue, thus ultrasound beam is reflected in form of artifacts known as B-lines Figure (1). B-lines belong to the family of the comet-tail artifacts commonly arising from gas densities within tissues examined by ultrasound $[4,5]$.

Pathological B-lines are thick and multiple , seen in parenchymal lung diseases Figure (2) and extend of B-lines corresponds to severity of involvement of lung parenchyma $[6,7]$.

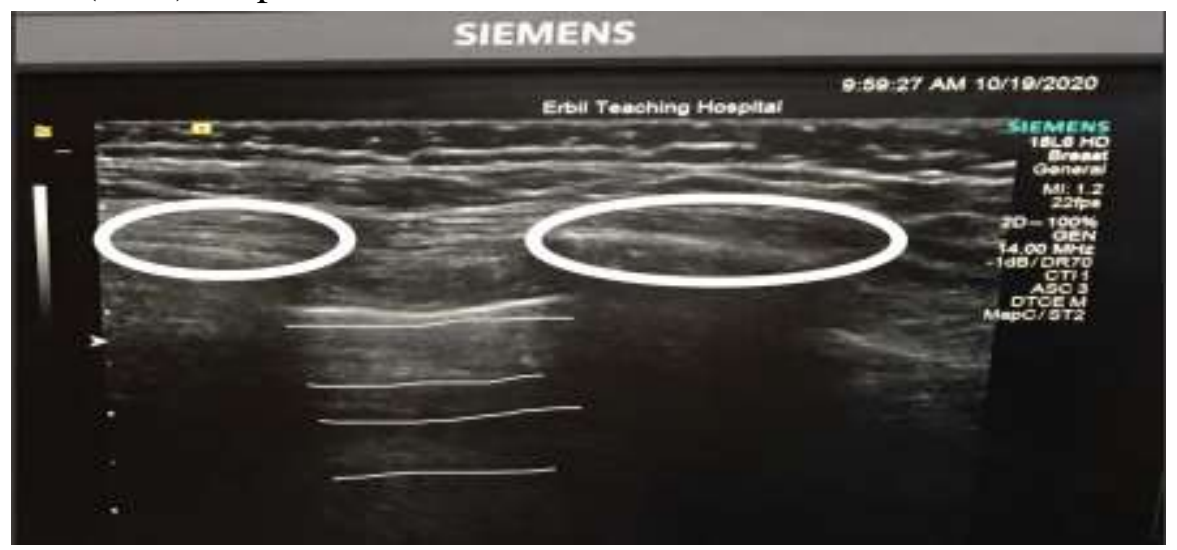

Figure (1): Normal lung appearance on ultrasound, thick white horizontal line is the normal smooth pleura visualized in the intercostal space, thin white lines are A- lines artifacts, white circles are bony ribs casting posterior black acoustic shadowing 
Advantage of Ultrasound: Absence of radiation / safe, low coast / affordable and repeatable , using portable ultrasound devices with advanced modern capabilities aids in lowering the contamination risk as the patient can be examined on bedside without moving the contagious patient to the radiology department $[8,9,10]$. The fact of no radiation exposure with LUS , make it suitably used for patient follow-up in particular for a pregnant patient infected with COVID-19 pneumonia $[9,10]$.

Limitation of US: LUS have lower sensitivity than CT as the ground glass may be difficult to detect by US . In addition the ground glass seen mainly in the posterior basal region that might be not easy to reach by sonographer due to the position of the severely ill patient in bed .US detect only peripheral subpleural changes, cannot detect deeper intrapulmonary abnormality and complications like pulmonary embolism. Moreover no panoramic views are possible in chest module ,along with large body habitus \& thick chest wall in patient with morbid obesity impairs lung parenchyma and pleural visualization as such presence of surgical emphysema and wounds with a tremendous amount of dressing [8].

Chest US manifestation in COVID-19 infection: $\mathrm{B}$-lines are a major feature of early stages which could be either discrete increase in number or confluent giving waterfall sign Figure (3) or white lung sign .Patchy consolidation Figure (6) is a less frequent sign which appears as a hypoechoic area deep to the pleura .Unsmooth rough pleural lines and sometimes air bronchograms[11] are the additional manifestations.

These findings are nonspecific can be seen in other viral infections, pulmonary edema, haemorrhage \& infarction, perhaps confirmation of the diagnosis requires complementary imaging modalities such as CT scan [10].

Chest HRCT manifestations in COVID -19 infection: Patchy ground glass opacities Figure (2) or consolidation Figures (4) and (5) or both are major manifestations, other less frequent findings are subpleural band, air bronchogram sign , architectural distortion, vascular cuffing and nodular opacities[13,14].
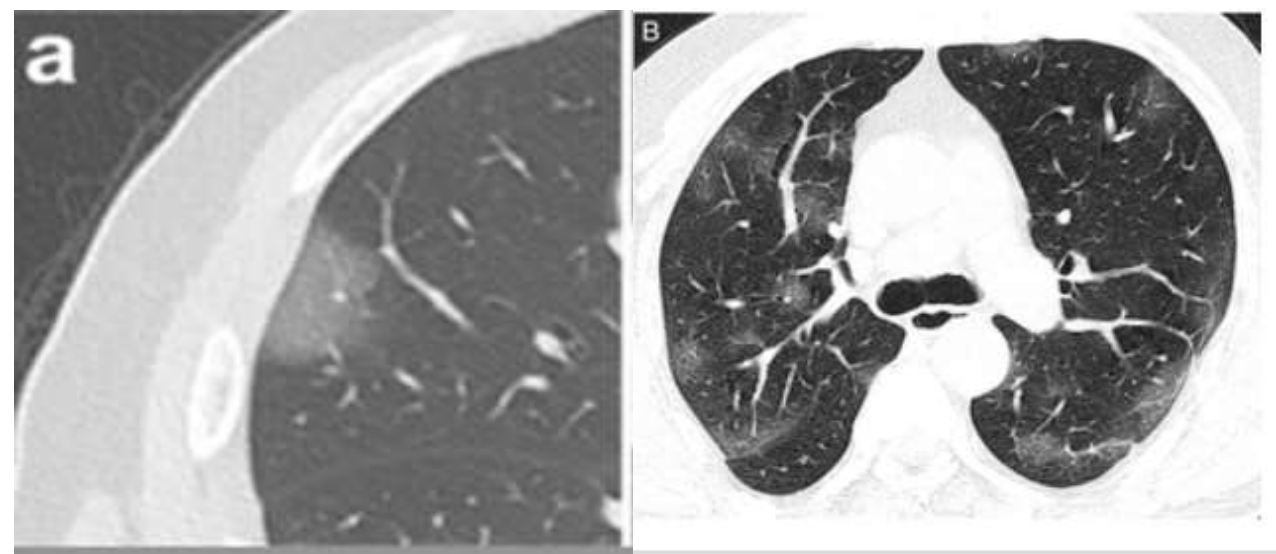

Figure (2): Appearance of lung parenchymal ground glass opacity on chest CT[12] 


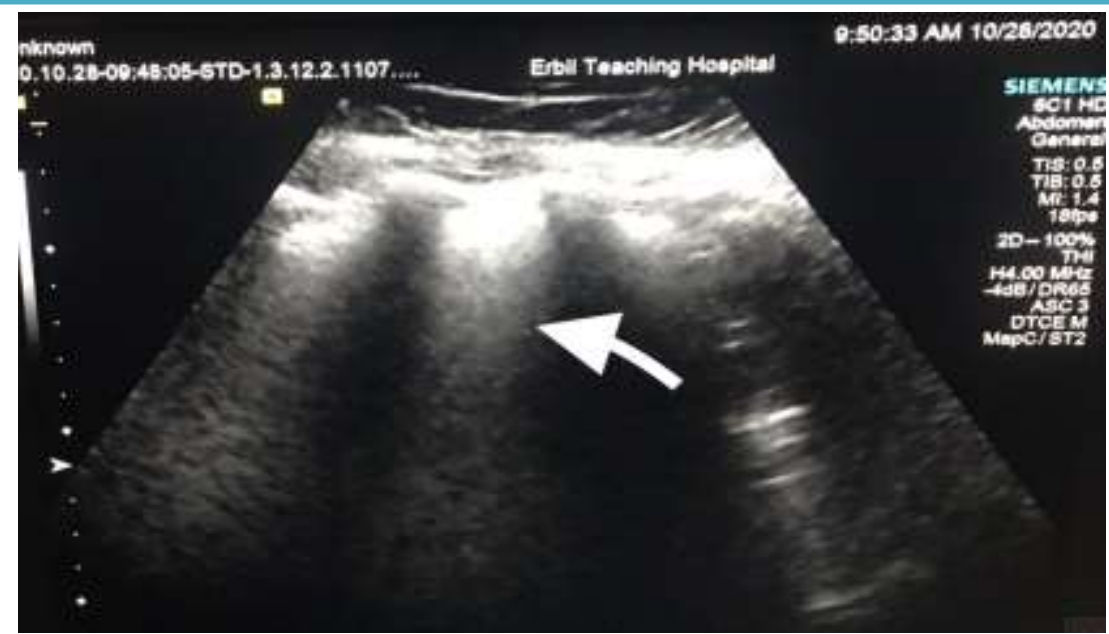

Figure (3): Axial view lung ultrasound, the white arrow indicats water fall sign due replaced air in the subpleural lung

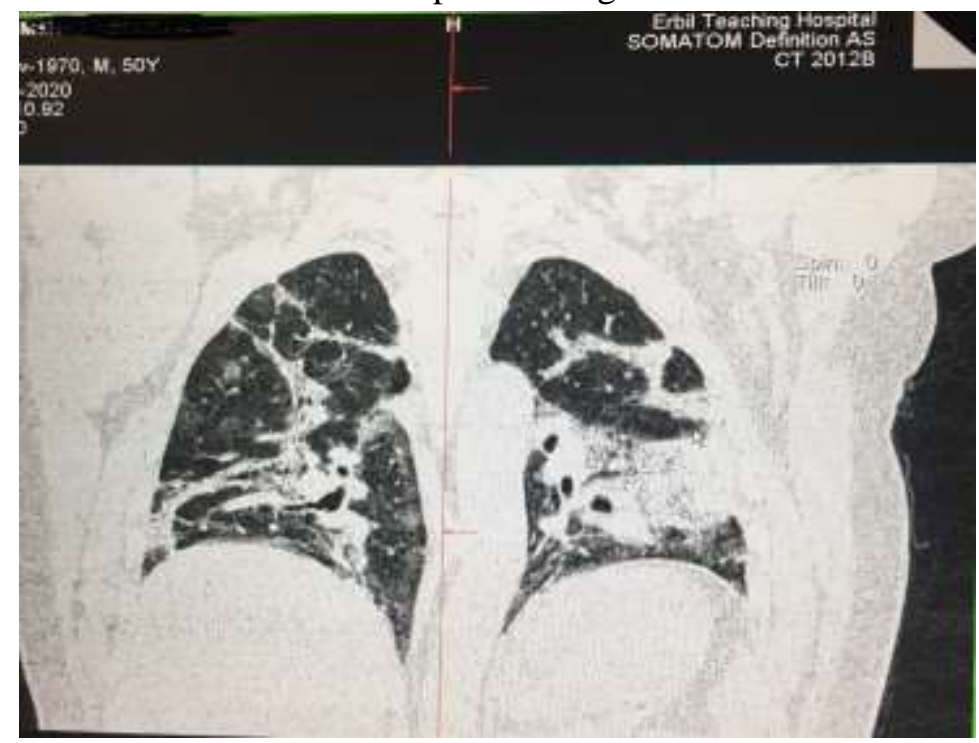

Figure (4) : Coronal reformate CT chest demonstrate pstchy conssolidation with air bronchogram sign

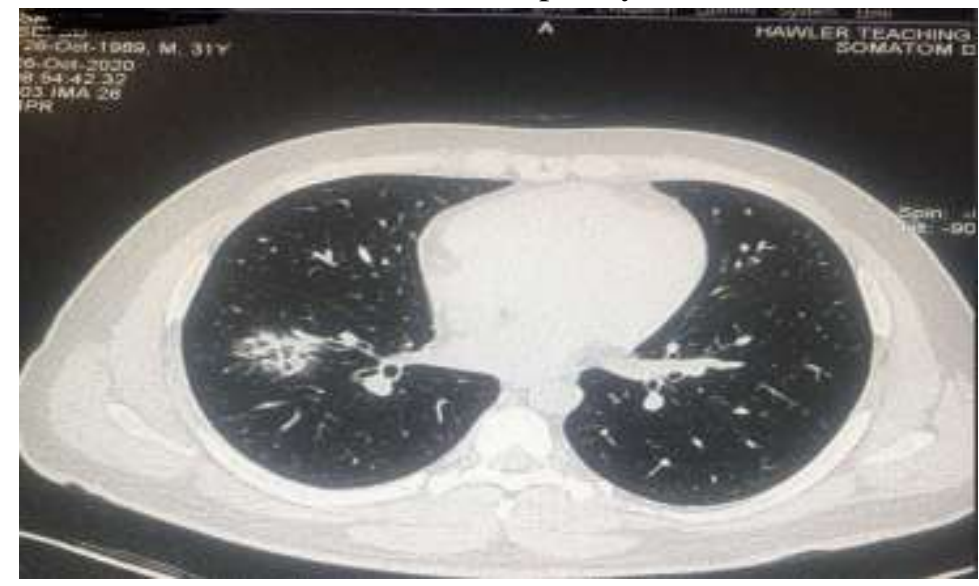

Figure (5) : Axial section CTchest pulmonary window shows patch of consolidation surrounded by rim of ground glass giving halo sign 


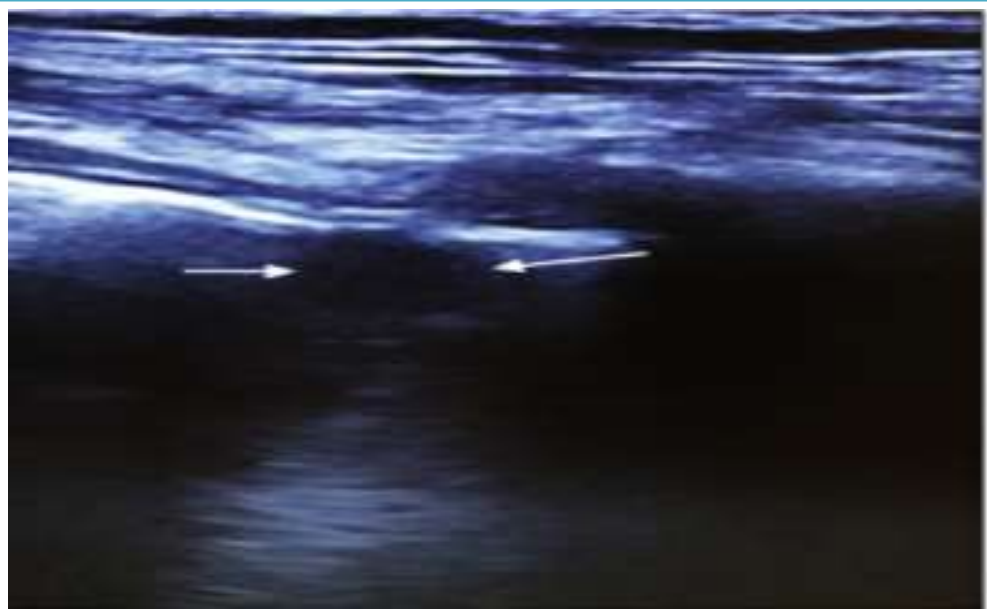

Figure (6): Ultrasound view of lung using linear prob showing consolidation between white arrows[12]

The aim of this study is to find the correlation between lung ultrasound and chest highresolution computed tomography (HRCT) signs in patients suffering from COVID-19 pulmonary involvement

\section{Patients and Methods}

This cross sectional single centre study conducted in Erbil city / Iraq, from July $10^{\text {th }}$ to November $1^{\text {st }} 2020$, Fifty consequent patients who referred to the radiology department-Erbil teaching hospital by medical specialists on the bases of high clinical suspicion of having various severity COVID-19 pneumonia, regardless of PCR status, evaluated with chest HRCT and targeted ultrasound examination of the chest done at the same time in the department. CT examination performed using multidetector 64 slice SIEMENS - SOMATOM 2012 machine, the individual parameters used- : KV 120 , eff .mAs 142 , ref. mAs 110 , slice thickness $0.75 \mathrm{~mm}$, reconstruction interval $1.0 \mathrm{~mm}$, the examination done with the patient in the supine position, no IV contrast used.

Scrollable consequent images analyzed and reported from the main screen, displayed width of 1200 Hounsfield unit (HU) and centre of $-600 \mathrm{HU}$ for the pulmonary window and width $320 \mathrm{HU}$ for mediastinal window used . The CT image appearance which looked for are :

-distribution of air spaces opacities (peripheral, central both )

-involved lung lobes in order of frequency (right upper, middle, lower lobes and left upper, lower lobes)

-involved lobes in numbers (one, two, three , four or five)

-pattern of lung involvement (ground glass opacity $+/$ - consolidation)

-additional signs (crazy paving pattern, air bronchogram sign, architectural distortion , band opacities, sub plural lines, vascular cuffing and nodular opacites)

-extra pulmonary manifestations (mediastinal enlarged lymph nodes and pleural effusion)

" The CT severity grades as described by the European Society of Radiology and European Society of Thoracic Imaging, used as follow: -grade 1 , absent or limited, with $<10 \%$ of the involved lung

-grade 2, moderate [10-25\%]

-grade 3, extensive [25-50\%] 
-grade 4, severe [50-75\%]

-grade 5, critical [>75\%] " [13]

Chest ultrasound examination performed using linear and convex transducers frequency ranging from 3 to $12 \mathrm{MHz}$ depending to the patient's body habitus (14) from SIEMENS / ACUSON system machine (S 2000), chest wall surface divided in to 12 definite zones, six lung regions on each side of landmarked by a parasternal, anterior axillary , posterior axillary , and paravertebral lines respectively as explained in Figure (7) $[15,16]$.

Pathological B-lines considered when 3 or more in number, thick or confluent detected in each examined zone were signed as Bscore 1. While the presence of consolidation in the viewed area was signed as $\mathrm{C}$-score 1 . The obtained total score from sum of B annd $\mathrm{C}$ was graded from 0 to 12 accordingly.

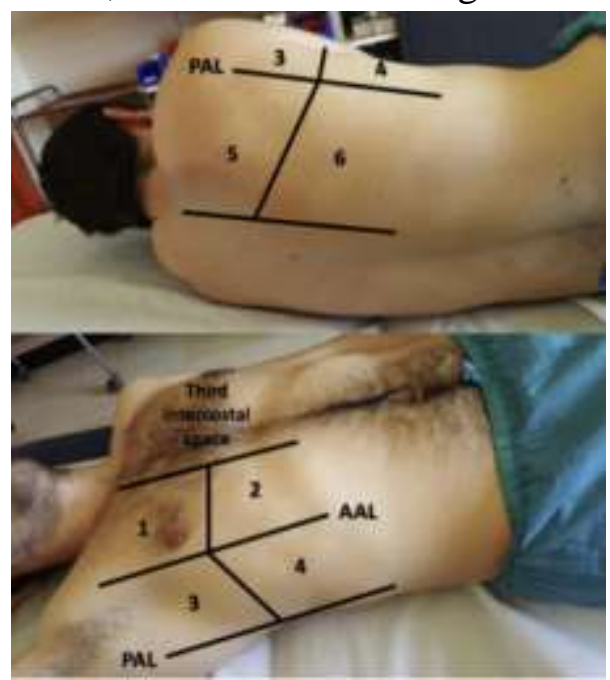

Figure (7) : Illustrate position of patient for examination , marking anatomical lines and zonal distribution in supine and lateral position. PSL, parasternal line; AAL, anterior axillary line; PAL, posterior axillary line. 1, anterosuperior region; 2, anteroinferior region; 3 , upper lateral region; 4, lower lateral region; 5, posterosuperior region; 6 , posteroinferior region [10]

\section{Statistical analysis}

Data obtained were presented in tables, correlation between CT and US score were assessed by Pearson correlation test using SPSS version 23 program, obtained $\mathrm{P}$ - value of $<0.05$ was regarded statistically significant. The sensitivity, specificity and accuracy are also calculated.

\section{Results}

This study includes 50 patients who referred by medical specialist for imaging, clinically highly suspecting to have COVID19 chest involvement, the LUS examination done retrogradely after positive chest CT, median age 65 [37-75] years , 33(66\%) male , 17(34\%) female. We assessed the radiological findings on HRCT, mixed ground glass seen in almost all patients 50 $(100 \%)$ while consolidation in 20 patient (40\%), distribution in peripheral and central seen in 30 patient $(60 \%)$, only centrally in 2 patient (4\%) and peripherally in 18 patient (36\%), most of the patient have right lower lobe involvement 45(90\%), then left lower lobe in $44(88 \%)$, left upper lobe $40(80 \%)$, 
right upper lobe $35(70 \%)$ \& right middle lobe 15(30\%) Table(1).

Most of patient 26 (52\%) have 4 lobes involvement, $20(40 \%)$ have 5 lobes involved, 10(20\%) 3 lobes involved, 13(26\%) 2 lobes involved and $4(8 \%)$ have 1 involved lobe.
Air bronchograms are the most specific sign detected in $16(32 \%)$ of patients, next crazy paving in $15(30 \%)$ other signs are less. The extrapulmonary manifestations were lymphadenopathy in 2(4\%) and pleural effusion in $1(2 \%)$.

Table (1): Chest findings on HRCT

\begin{tabular}{|c|c|c|}
\hline Lesion & Number & $\%$ \\
\hline $\begin{array}{cl}\text { Distribution : } \\
\text { - } & \text { Central } \\
\text { Lobe: } & \text { Poth } \\
\text { - } & \text { Right upper } \\
\text { - } & \text { Right middle } \\
\text { - } & \text { Right lower } \\
\text { - } & \text { Left upper } \\
\text { Number of involved lobes: } \\
\text { - } & 1 \\
\text { - } & 2 \\
\text { - } & 3 \\
\text { - } & 4 \\
\text { Appearance: } \\
\text { - } & \text { Ground glass opacity } \\
\text { - } & \text { Consolidation } \\
\text { Specific additional signs: } \\
\text { - } & \text { Crazy paving } \\
\text { - } & \text { Air bronchogram } \\
\text { - } & \text { Atoll sign } \\
\text { - } & \text { Architectural distortion } \\
\text { - } & \text { Subpleural line } \\
\text { - } & \text { Nodular opacity } \\
\text { Extrapulmonary manifestation: } \\
\text { - } & \text { Lymphadenopathy } \\
\text { Pleural effusion }\end{array}$ & $\begin{array}{c}4 \\
13 \\
10 \\
26 \\
20 \\
\\
50 \\
20 \\
\\
15 \\
16 \\
10 \\
2 \\
20 \\
35 \\
2\end{array}$ & $\begin{array}{c}8 \\
26 \\
20 \\
52 \\
40 \\
\\
100 \\
40 \\
\\
30 \\
32 \\
20 \\
4 \\
40 \\
70 \\
4\end{array}$ \\
\hline
\end{tabular}

In this study the most common percentage lung involvement scoring on $\mathrm{CT}$ was moderate seen in 20(40\%), extensive seen in
$13(26 \%)$, severe in $10(20 \%)$, limited in $4(8 \%)$ and critical in $3(6 \%)$ patients , average lung injury was $40 \%$ Table (2). 
Table (2): chest findings on HRCT

\begin{tabular}{||l||c||c|}
\hline \multicolumn{1}{|c|}{ CT severity } & Number & $\%$ \\
\hline Limited or absent $<10 \%$ & 4 & 8 \\
Moderate $10-25 \%$ & 20 & 40 \\
Extensive 25-50\% & 13 & 26 \\
Severe 50-75\% & 10 & 20 \\
Critical > 75\% & 3 & 6 \\
Average \% score 40\% & & \\
\hline
\end{tabular}

Regarding the most prevalent pattern of lung involvement detected on US was B-line (B) which seen in $37(74 \%)$, consolidation $(C)$ in
$4(8 \%)$ and both (B and C) in 9(18\%), ancillary findings like pleural effusion and adenopathy seen only in 3(6\%), Table (3).

Table (3) : Ultrasound pattern of lung involvement

\begin{tabular}{||l||c|c||}
\hline \multicolumn{1}{|c||}{ Pattern } & Number & $\%$ \\
\hline \hline patients with B-lines & 37 & 74 \\
patients with consolidation & 4 & 8 \\
patient with both & 9 & 18 \\
patient with additional findings & 3 & 6 \\
\end{tabular}

The US scoring obtained by adding the findings in all 12 areas in bilateral chest depict that most of the examined patients have score between 3-6 corresponds to moderate degree seen in $16(32 \%)$, while
$15(30 \%)$ have score between 6-9 and 8(16\%) have lowest score $<3$ and only 11(22\%) have high score $>9$, The mean score of 12 evaluated zones by LUS was 7 (6-9) illustrated in Table (4).

Table (4) : Ultrasound lung severity scoring by summation of 12 area of chest examination

\begin{tabular}{||c||c||c||}
\hline \multicolumn{1}{|c||}{ Score } & Number & $\%$ \\
\hline \hline$<3-6$ & 8 & 16 \\
$6-9$ & 16 & 32 \\
$>9$ & 15 & 30 \\
\hline
\end{tabular}

There was a significant correlation among findings obtained by both imaging modalities , and obvious association found between LUS severity scores and the extent of lung parenchymal involvement seen on CT, thus the more US scores the more extensive lung involvement detected by CT scan $(r=0.78$, $\mathrm{p}<0.001)$ Figure (8). 


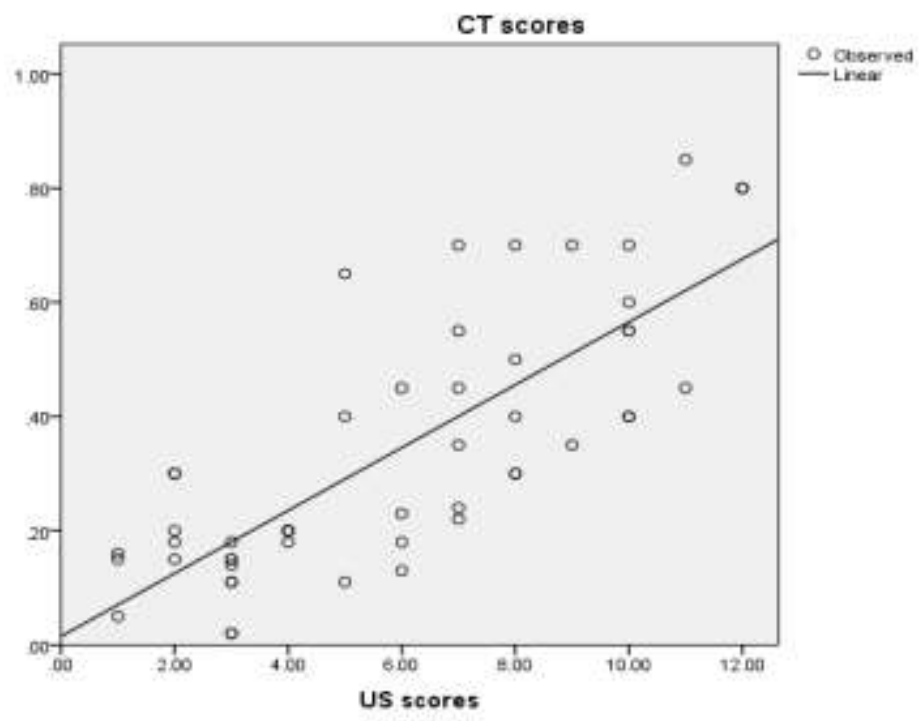

Figure (8): The correlation between LUS aeration score and percentage of lung involvement detected on CT

Sensetivity of LUS was $90 \%$, specificity $70 \%$

and accuracy $68 \%$.
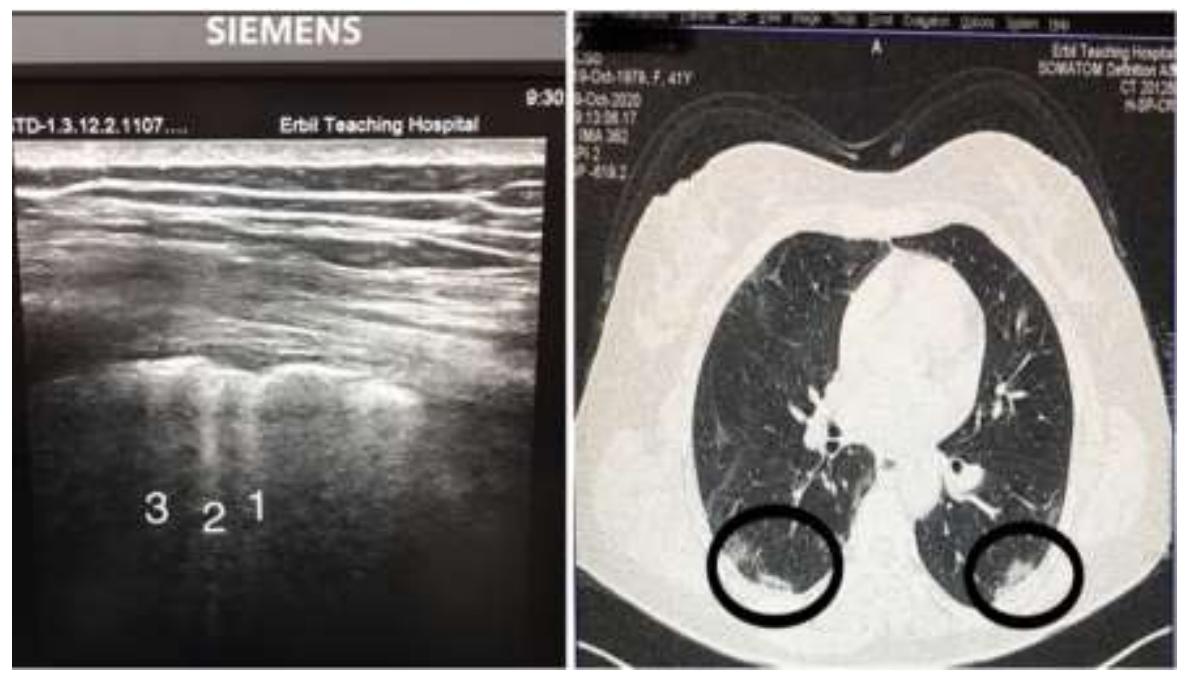

Figure (9) :Chest US and reciprocal CT images in patient with COVID-19 pneumonia, correlation between the findings, image A: multiple B-lines (numbered) with irregular pleural line on US, image B: CT peripheral subpleural ground glass opacities (black circles) obtained at same time, same area and

\section{Discussion}

Chest imaging plays a crucial role in the diagnostic workup for evaluation of patients with suspected COVID pneumonia[17]. In this context, there is more increasing

utilization of LUS as the simplest tool for diagnosis and screening of viral pneumonia, the fact that air space opacification due to pneumonia localized predominantly in the 
periphery, this makes detection by LUS easier [22,23].

A study done in Guangdon / south china by $\mathrm{Lu}$ et al team found that the diagnostic accuracy was of $(93.3 \%)$, sensitivity of $(100.0 \%)$, and specificity of $(85.7 \%)$ of bedside LUS which considered very high for severe lung lesions in COVID-19 patients. However, the obtained parameters was low for mild degree of lung involvement in term of sensitivity of $(68.8 \%)$, specificity of $(85 \%)$, diagnostic accuracy of(76.7\%) while for moderate degree of lung lesions were as follow :sensitivity of(77.8\%), specificity of $(76.2 \%)$, diagnostic accuracy of $(76.7 \%)[10]$. However in our study the overall parameters were : Sensitivity of $(90 \%$ ), Specificity of $(70 \%)$ and Accuracy of(68 $\%)$ for point of care lung US in the selected sample regardless the degree of severity.

According to another study done by Sebastian et al in Paris / France, the obtained severity of lung involvement by COVID-19 pneumonia on chest HRCT were: absent or limited disease in 2 patient $(9.5 \%)$, moderate disease in 10 patient (48\%), extensive lung involvement in 5 patient ( $24 \%$ ), severe lesion in 4 patient (19\%) and no patient have critical lung involvement, with mean percentage of lung involvement was $20 \%$ [25].The currently conducted study reveals commonest extend of lung involvement on HRCT was: moderate seen in $20(40 \%)$, extensive seen in $13(26 \%)$, severe in $10(20 \%)$, limited in $4(8 \%)$ and critical in $3(6 \%)$ patients in order of frequency and the average lung lesions was $40 \%$.

At least 19 patient $(90.5 \%)$ have an area of pathological B- lines detected on LUS and 13 patient (61.9\%) shows consolidation lesion by LUS obtained in study done by Sebastian et al [25].In our study the pathological B-line (B) was seen in 37(74\%) , consolidation (C) in 4(8\%) and both (B)and (C) in $9(18 \%)$, ancillary findings like pleural effusion and adenopathy was seen only in $3(6 \%)$. A total agreement was noted upon the correlation of LUS score with chest HRCT severity percentage of current study compared to that obtained in same study done in France which was $(r=0.935, \mathrm{p}<0.001)$ [25] and our result which was $(\mathrm{r}=0.78$ and $\mathrm{P}$ $<0.001)$.

On the other hand this study results closely matched to another done in Ro de janeiro / Brazil by Agnaldo et al, in which 45 hospital admitted COVID-19 pneumonia patients examined by LUS followed by chest HRCT within 24 hours after LUS examination, they found that $17(37.8 \%)$ were men, and 28 $(62.2 \%)$ were women, mean age of 44 years , LUS viral pneumonia signs in term of pathological B-lines seen in $73.3 \%$ and consolidations seen in $24.4 \%$ of cases, while CT ground glass opacities observed in $(66.7 \%)$, the crazy-paving in (20\%) parenchymal bands in $(17.8 \%)$ cases , consolidations, the halo sign, and subpleural lines were observed in $(20 \%),(15.6 \%)$, and (6.7\%) cases respectively. Regarding the relation between the LUS score and the chest CT percentage involvement, patients who had a more LUS scores expressed more severely involved lung on corresponding CT examamination [21].

LUS score have appreciable relation to severity score obtained by HRCT that's concluded in a study done in Parma/Italy by Nouvenne, in term of statistical data $(r=$ $0.65, \mathrm{p}<0.001)$,additionally their 
conducted study showed a higher extent of HRCT severity score in patients with LUS scores above average than patients with LUS scores below average $(\mathrm{p}=0.016)$, as they divided LUS score patients in to 2 groups : above and below average [24].That's also parallel to results obtained in our study .

Like other works, this study have some limitations, first is selection bias, as the patients are exclusively those having positive findings on $\mathrm{CT}$ chest were assessed by targeted LUS scan.Virtual limitations of LUS despite several advantages over other imaging modalitie, it is poor in detection of lung lesions deep to pleural level because air filled lung prevent sound wave penetration [8].And lastly LUS is more operator dependent, needs dedicated experience and training, therefore confirmation of LUS findings with CT scanning seems advantageous.

\section{Conclusions}

Thus conclusively we believe that LUS can be implemented as CT alternative specially when planning a suitable diagnostic workup using least expensive available technological resources particularly in the setting of pandemic crises, perhaps HRCT regarded as standard reference modality for screening and diagnosing pneumonia in COVID-19 suspected cases, having additional advantage in excluding underlying complications like pulmonary embolism, as thromboembolic phenomena recently found to be of great risk in COVID-19 victims [26].

\section{Recommendations}

Our study includes small number of patients, so recommended for adequately larger scale studies for establishing diagnostic accuracy of chest sonography in patient with COVID-19 pneumonia in term of sensitivity and specificity in addition assessing response after treatment. Furthermore recommend the utility of this useful , commonly available and cheap imaging modality to be extended beyond this pandemic towards more diagnostic usefulness.

Source of funding: No special funding as the study done in public hospital.

Ethical clearance: From college of medicine / Hawler medical university.

Conflict of interest: No disclosure or interested conflict.

\section{References}

[1]Mechael C, Adam B, Xueyan. M, Ning Z, Mingqian $\mathrm{H}$, Xianjun $\mathrm{Z}$ etal.CT imaging features of 2019 novel coronavirus (2019nCoV) Radiology, 295 (1) ;2020 . p. 202-7. [2]Tao A, Zhenlu Y, Hongyan H, Chenao Z, Chong C, Wenzhi Lv, et al.Correlation of chest CT and RT-PCR testing in coronavirus disease 2019 (COVID-19) in China: a report of 1014 cases Radiology ;2020 . p. 200642. View Record in ScopusGoogle Scholar. [3]Smith M.J, Hayward S.A, Innes S.M , Miller A. Point-of-care lung ultrasound in patients with COVID-19-a narrative review Anaesthesia, 75 (8) ;2020 Aug · p. 1096-04. [4]Ziskin MC, Thickman DI, Goldenberg NJ, Lapayowker MS, Becker JM: The comet tail artifact. J Ultrasound Med $1982 ; 1: 1-7$.

[5]Volpicelli G, Elbarbary M, Blaivas M, Lichtenstein DA, Mathis G, Kirkpatrick AW. International Liaison Committee on Lung Ultrasound (ILC-LUS) for Interna tional Consensus Conference on Lung Ultrasound (ICC-LUS). International evidence-based 
recommendations for point-of-care lung ultrasound. Intensive Care Med 2012; 38:577-91.

[6]Gargani L, Picano E, Caramella D, Abramo A, Giunta F, Forfori F. Lung water assessment by lung ultrasonography in intensive care: a pilot study. Intensive Care Med 2013; 39:74-84.

[7]Soldati G, Copetti R, Sher S: Sonographic interstitial syndrome: the sound of lung water. J Ultrasound Med 2009; 28:163-17

[8]Peng Q-Y, Wang X-T, Zhang L-N, Chinese critical Care Ultrasound Study G. Findings of lung ultrasonography of novel corona virus pneumonia during the 20192020 epidemic. Intensive Care Medicine; 2020 .

[9]Bounsenso D, Piano A, Raffaelli F, Bonadia N, Donati K, Franceschi F. point of Care Lung Ultrasound findings in novel coronavirus disease -19 pneumonia: a case report and potential application during COVID-19 out brake, European review for medical and pharmacological sciences .2020; 24(5):2776-80.

[10] Lu W, Zhang S, Chen B, Chen J, Xian J, Lin Y, et al. A clinical Study of Non invasive Assessment of Lung lesions in Patients with Coronavirus Dsisease-19(COVID-19) by Bedside Ultrasound. Ultraschall in Med.2020 (EFist)

[11]Reissig A, Copetti R, Mathis G,Christin $\mathrm{M}$, Andreas $\mathrm{S}$, Peter $\mathrm{Z}$, et al. Lung ultrasound in the diagnosis and follow-up of community-acquired pneumonia: a prospective, multicenter, diagnostic accuracy study. Chest 2012; 142:965-72.

[12]Xia Y, Ying Y, Wang S, Li W, Shen H. Effectiveness of lung ultra- sonography for diagnosis of pneumonia in adults: a systematic review and meta-analysis. J Thorac Dis 2016; 8:2822-2831 [13]Marie-Pierre R , Parkar AP , Prosch H ,Mario S ,Nicola S ,Fergus G ,et al.COVID19 patients and the radiology department advice from the European society of radiology (ESR) and the European society of thoracic imaging (ESTI) Eur Radiol ;2020 Apr 20 . p. 1-7

[14]Gino S, Andrea S, Riccardo I, Danilo B , Tiziano P, Domenica F,et al. Proposal for international standardization of the use of lung ultrasound for patients with COVID-19: a simple, quantitative, reproducible method $\mathrm{J}$ Ultrasound Med, 39 (7) ;2020 Jul .p. 141319.

[15]Belaid B. Helen B, Morgan L, Charlotte L, Qin L, Jean-Jaques R .Bedside ultrasound assessment of positive end-expiratory pressure-induced lung recruitment Am J Respir Crit Care Med, 183 (3) ;2011 .p. 34147.

[16]Perera P, Mailhot T, Riley D, Deku M. The RUSH exam: Rapid Ultrasound in SHock in the evaluation of the critically ill. Emerg Med Clin North Am. 2010; 28:29-56, vii. [ Links ]

[17]Hun Jung K, Soyeoun L, Jooae C,SangHo C, Heungsub S, Kyung Hun D et al. Radiographic and CT features of viral pneumonia Radio Graphics, 38 (3);2018 . p. 719-739

[18]Fabio G, Francesco F,Guarracino F, Vetrugno L, Bove T,Copetti R et al. Lung, heart, vascular , \&diaphragm Ultrasound examination of COVID-19 patients, Jul 2, 2020 : A comprehensive Approach. Available at https://www.jcvaonline.com/article/S1053$\underline{0770(20) 30519-X / a b s t r a c t}$ 
[19]Moro F, Buonsenso D, Moruzzi MC, Inchingolo R, Smargiassi A, Demi L et al . How to perform lung ultrasound in pregnant women with suspected COVID-19. Ultrasound Obstet Gynecol. 2020 May;55(5):593-598. doi: 10.1002/uog.22028 [20]Volpicelli G,Elbarbary M ,Blaivas M , Lichtenstein D.A ,Mathis G., Kirkpatrick A , et al , International Liaison Committee on Lung Ultrasound (ILC-LUS) for the International Consensus Conference on Lung Ultrasound (ICC-LUS), International evidence-based recommendations for pointof-care lung ultrasound, Intensive Care Med. 2012; 38: 577-591

[21]Agnaldo $\mathrm{J}$,Thiago $\mathrm{T}$, Cláudia $\mathrm{H}$, Rogério R , Mônica C , Kennedy K, et al , Comparison Between Lung Ultrasound and Computed Tomographic Findings in Patients With COVID-19 Pneumonia, available at https://onlinelibrary.wiley.com/doi/full/10.10 $\underline{02 / j u m .15521}$

[22] Yang Y, Huang Y, Gao F, Yuan L, Wang Z. Lung ultrasonography versus chest $\mathrm{CT}$ in COVID-19 pneumonia: a two-centered retrospective comparison study from China [published online ahead of print May 25, 2020]. Intensive Care Med. 2020; 46: 17611763. https://doi.org/10.1007/s00134-02006096-1.

[23]Dong D, Tang Z, Wang S, et al. The role of imaging in the detection and management of COVID-19: a review [published online ahead of print April 27, 2020]. IEEE Rev Biomed Eng. https://doi.org/10.1109/RBME.2020.2990959 [24]Nouvenne A, Zani MD, Milanese G, et al. Lung ultrasound in COVID-19 pneumonia: correlations with chest $\mathrm{CT}$ on hospital admission. Respiration 2020; 99: 617-624

[25]Sebastian O, France M ,Ebistien E, Luci D, Corali L, Mari Pier D et al. Lung ultrasonography in patients with COVID-19: comparison with $\mathrm{CT}$, available at https://doi.org/10.1016/j.crad.2020.07.024Ge t rights and content [26] Klok F, Kruip M, Van deer N, Arbus M, Gommer $\mathrm{D}$, Kant $\mathrm{K}$ et al .Incidence of thrombotic complications in critically ill ICU patients with COVID-19 Thromb Res, 191 ;2020 Jul . p. 145-147. 